\title{
The design of a double ended interferometer (DEI)
}

\author{
O. Kruger ${ }^{1, \star}$, F. Hungwe ${ }^{1}$, N. Farid ${ }^{2}$, and K. Schreve ${ }^{3}$ \\ 1 NMISA, Private Bag X34, Lynwood Ridge 0040, Pretoria, South Africa \\ 2 NIS, Tersa st., Haram, Giza code 12211, P.O.136, Giza, Egypt \\ 3 SUN, Private Bag X1 Matieland 7602, South Africa
}

Received: 24 March 2014 / Accepted: 3 October 2014

\begin{abstract}
At NMISA the SI unit for length is realised by an iodine stabilised He-Ne laser, an optical measurement. In industry most measurements are performed by mechanical probing. Gauge blocks are the link between the optical measurements and the mechanical measurements, and are therefore critical in disseminating traceability. The gauge block length is currently determined by wringing the gauge block onto a platen. This is laborious, requires skill and causes contact errors. Since around 1943, there have been efforts to build a double ended interferometer where gauge block length can be determined without wringing it onto a platen. While there has been a lot of progress in building DEIs, to date, there is still no commercially available DEI. We present a collaboration project between the National Metrology Institute of South Africa (NMISA), the National Institute of Standards (NIS Egypt) and Stellenbosch University (SUN) in South Africa to build a double ended interferometer for use at the respective National Metrology Institutes. We investigate improvements to the interferometric calibration of gauge blocks and recent developments. The different systems currently in use in the national metrology laboratories are described and various designs are investigated. Lastly the expected outcome of the project will be discussed.
\end{abstract}

Keywords: Gauge blocks, length bars, interferometer

\section{Introduction}

The need for accurate length measurements and definition is needed throughout the modern world. Much of industry and technology relies on these measurements. From the thread on a nut and bolt to precision machined parts in car engines down to tiny structures on micro-chips, all require an accurate international scale of length. This need is all the more important in the global economy, because without it, for example, engine parts made in South Africa would not fit an engine in the United Kingdom.

The 1927 the SI unit of length was defined by an optical standard. Up to then the metre was defined by the line standard that was kept by the BIPM and to which National Metrology Institutes, NMIs around the world compared theirs against.

As this was a physical standard, the risk of damage, drift and better uncertainty, a new definition was researched and first it was the wavelength of Cadmium, which was later replaced by the wavelength of Krypton.

On 20th October 1983, at the 17th General Conference on Weights and Measures the new definition for the metre as "the length of path travelled by light in vacuum during a time interval of (1/299 792 458) of a second" was adopted.

Up to recently, the most common practice, as is the case in South Africa to realise the definition for the metre,

* Correspondence: oakruger@nmisa.org is through the wavelength of the $632,8 \mathrm{~nm}$ radiation of an Iodine stabilized Helium-Neon laser (Fig. 4). The reproducibility is about 3 parts in $10^{--11}$ equivalent to measuring the earth's mean circumference at the equator to about $1 \mathrm{~mm}$.

However, research over the last few years into frequency combs lead to more NMIs establishing frequency combs as their national standards for length.

Traceability from the national for length are through a traceability chain from the Iodine stabalised laser to secondary lasers interferometers to the calibration of gauge blocks as in Figure 1, traceability chart. Up to this point all measurement are performed by optical methods.

However measurements in industry are performed by mechanical probing. Gauge blocks are the link between the optical measurements to the mechanical probing measurements eg. diameters, calibration of hand tools like verniers and micrometers and length measuring instruments.

\section{Calibration of gauge blocks}

So although gauge block calibration are well established and performed over a 100 years, it is still critical ion the traceability chain from the SI definition of the metre to the measurement performed by industry on the shop floor as shown in Figure 1.

The standard, ISO 3650 [3], in which the calibration and specification of gauge blocks is described, the length 


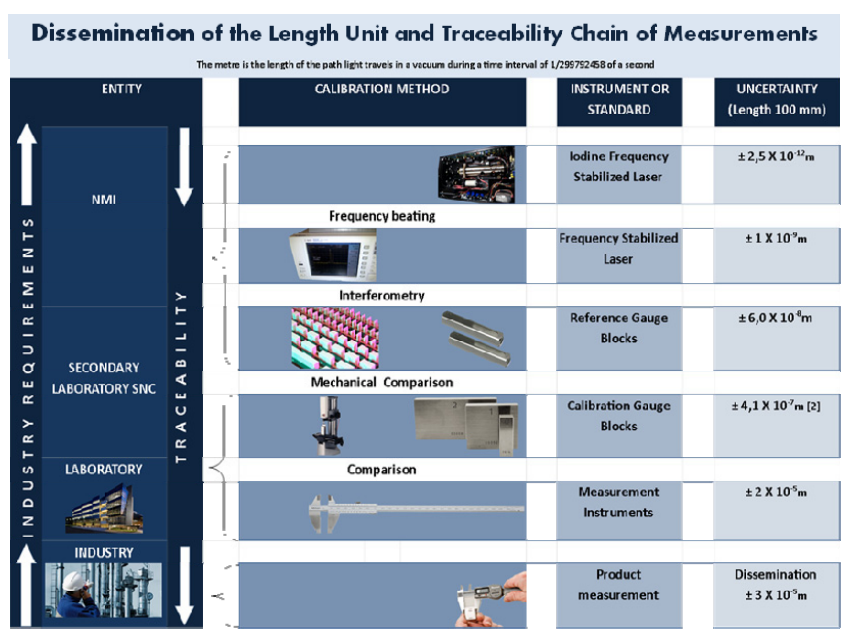

Fig. 1. Traceability chart from national standard to industry.

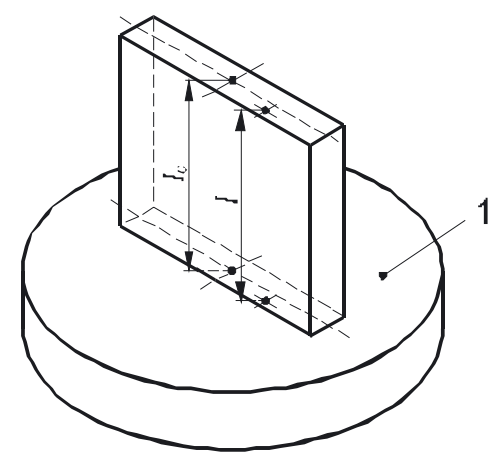

Fig. 2. Centre length lc of a gauge block wrung to the plane surface of an auxiliary plate [3].

of a gauge block is defined as: "the perpendicular distance from any particular point on the gauge block and a plane surface of an auxiliary plate of the same material and surface texture upon which the measuring face has been wrung". With a special note: "This length of the gauge block, 1 , includes the effect of one-face wringing". This set-up is shown in Figure 2.

Apart from the general conditions, the standard further prescribes that the centre length of the measuring face shall be measured using the method of interferometry. The auxiliary plate shall be of the same material as the gauge block and have a wringing surface similar to the surface finish of the measuring face of the gauge block. If auxiliary plates of other materials are used then corrections must be made.

\section{Difficulties and restrictions with the calibration of gauge blocks and length bars}

Although the calibration of gauge blocks is well defined in the ISO standard, many difficulties exist with these measurements and these difficulties are the reason why no real improvement has been achieved in the uncertainty of the measurements over the last 100 years.
The use of a platen to wring gauge blocks causes errors which contribute to uncertainties. Many studies made by Siddall, some as far back as 1969 [4], investigate the error sources focusing on the wringing between the gauge block and the platen. Many thereafter [5-7] continue this research. These studies discuss the effects of the difference in surface texture between the gauge block and platen and the phase corrections due to the different materials of the two. All these concluded that there is a significant contribution to the total uncertainty due to this wringing film and phase correction, with a deviation of up to $18 \mathrm{~nm}$. Taking this into account the expanded uncertainty for calibrating gauge blocks at NMISA is $23 \mathrm{~nm}$.

Other investigations, in particular, by Titov in various papers $[8,9]$, explore the measurements of gauge blocks to sub $\mathrm{nm}$ and suggested ways to overcome this phase change deviation. One of the suggestions was wringing with a slave block technique. This suggestion was questioned by Bonsch [10] where he refers back to the ISO standard which includes the use of the platen which was not correctly applied in these papers by Titov. Bonsch, in a different paper, investigated different techniques where the use of an integrating sphere is employed to measure the phase correction [11]. He proposes that every gauge block must be measured separately and a correction applied. With this method, the contribution from the wringing film can be reduced to less than $10 \mathrm{~nm}$.

Apart from the difficulty in phase correction between the gauge block and the platen, the wringing itself is also a major concern. The gauge block has to be wrung every time it is calibrated and with a calibration interval of between 1-5 years, a gauge block can end up being wrung more than 20 times in its life time. This will affect the length of the gauge block as described by Siddall. He shows that wringing gauge blocks 50 times can result in a wringing variation of as high as $20 \mathrm{~nm}$ for steel gauge blocks.

Another difficulty with the calibration of gauge blocks is that, the wringing is very time consuming and it is difficult to get a "good" wring where the gauge block is wrung flat and parallel to the platen, especially for gauge blocks smaller than $5 \mathrm{~mm}$ in length.

\section{Current systems in use at NMISA for the calibration of gauge blocks and length bars}

At NMISA, gauge blocks are calibrated using the TESA [12] gauge block interferometer. The interferometer can measure gauge blocks up to $300 \mathrm{~mm}$ in length. The system was designed to use platens made out of steel, tungsten carbide (TC) or ceramic, depending on the gauge block being calibrated.

Because gauge blocks of imperfect geometry and those smaller than $5 \mathrm{~mm}$ are difficult to wring, and one cannot determine if a "good" wring has been achieved, we use quartz platens for all gauge blocks. An in house study of this practice showed that the use of quartz platens can 


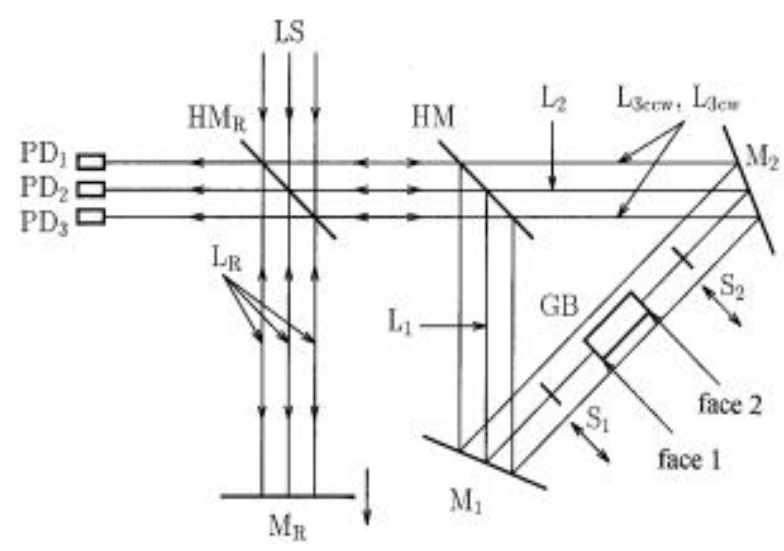

Fig. 3. Optical layout by Ishii [1].

be used in the calibration of gauge blocks with a good uncertainty due to the better wringing compared to the conventional steel platens as long as a phase correction is applied for the difference in material between the gauge block, steel and the platen, quartz.

The system for the measurement of long series slip gauges/length bars was based on a white light interferometer and, like the gauge blocks interferometer, the length bars had to be measured with one face wrung to a platen as described in the ISO standard. However the majority of length bars received for calibration was of a poor quality and therefore, not good enough to be wrung to a platen as they damage the platens [13]. Because of this, a system was developed to measure the length bars with a probing system where no wringing is necessary [14]. This system was fit for purpose at the time but with new measuring machines for example the ZEISS CMM being acquired, a higher accuracy of length bar calibration is required.

\section{Different systems investigated}

Taking all the difficulties mentioned above into account, and that there is no commercially available DEI, we decided to design and build one. The final system must able to measure without wringing onto a platen. It also must be able to measure both gauge blocks and length bars up to $1 \mathrm{~m}$. However, our initial design is for small lengths.

We looked at the double ended interferometer that has already been designed and built by Abdelaty A., and the other system designed by Ishii Y (Figs. 3 and 4). We also looked at a third system [15] that uses a white light interferometer but it is beyond the scope of our work.

The following figures show the different layouts of two of the system.

Whilst both designs are good, we decided to base our design on the layout by Ishii et al. The main reason is that this system has less optical components and is therefore cheaper and has a simpler design. If however we find this design challenging, we can easily add or remove components and experiment with the second design.

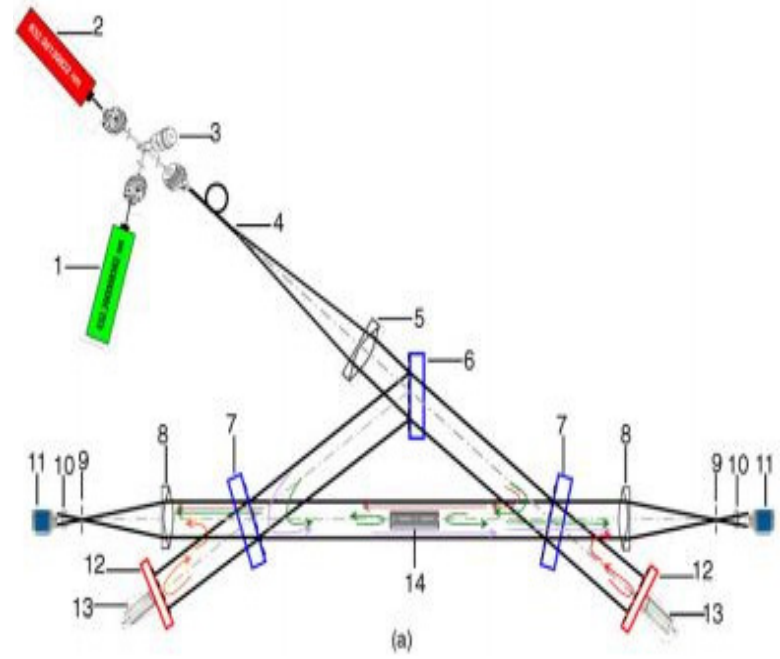

Fig. 4. Optical layout of system used by Abdelaty [2].

\section{NIS approach and work to date}

The calibration of short gauge blocks (range between $0.5-100 \mathrm{~mm}$ ) is carried out at National Institute for Standards (NIS) in Egypt by interferometry using a Koster comparator. This interferometer is an outdated model, and was one of the first wave equipment's granted to NIS by UNISCO. In case of calibration of longer gauge blocks (range up to $1 \mathrm{~m}$ ), mechanical probing techniques are used (e.g. CMM). Calibration method and result analysis of the static interferometer "Koster" is complicated and requires time. This makes the calibration costly to most of the sectors. On the other hand, recent industrial applications require very accurate results for the products of tightened tolerance, and mechanical calibration using probing techniques (i.e. CMM and mechanical comparator) may not be suitable for such applications.

Since the equivalence to the NMI's emphasize on high accurate measurements with low uncertainty, it has become necessary for NIS to enhance the calibrations procedures either by purchasing a modern interferometer (which is very costly) or constructing its own system depending on: the desired calibration method, range, and analysis procedure.

The bilateral project gives the opportunity to Egyptian and South African metrologists to practice and exchange knowledge in this field.

The NIS is to construct the interferometer as shown in Figure 6 to avoid any contact to the artifact surface through wringing to substrate. The next step is to investigate the use of the phase shifting method to study the whole surface of the artifact instead of single point measurement and to simplify the fringe analysis as it does not require the location of fringes centers.

A simple prototype for phase shifting technique is constructed in the preliminary experimental work to examine a surface of a gauge block. The existing facilities enable shifting of the object for $\pi / 2$.

Three images are recorded: $I_{\mathrm{O}}$ without phase shift, $I_{\pi / 2}$ with phase shift $\pi / 2$, and $I_{\pi}$ with phase shift $\pi$. 


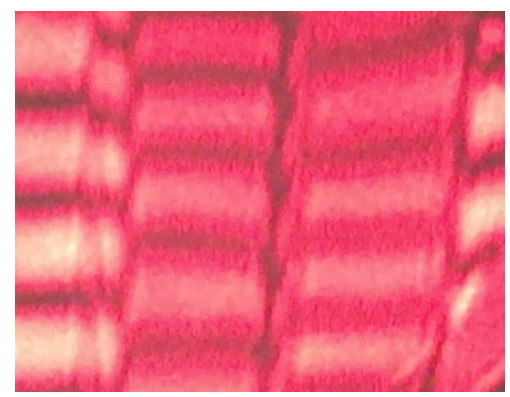

Fig. 5. Fringes from two gauge blocks and from the interferometer.

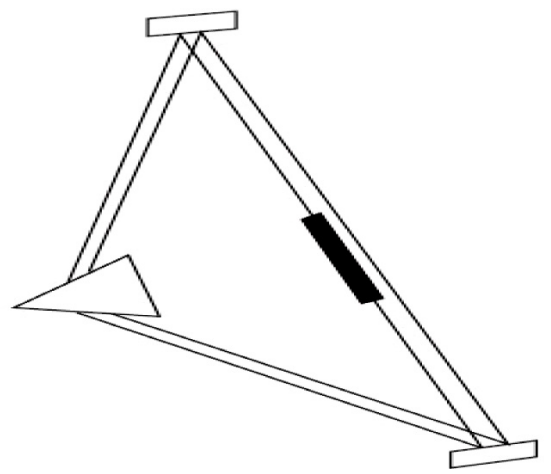

Fig. 6. NMISA design of the Double ended gauge block interferometer.

The corresponding intensities are given by:

$$
\begin{array}{r}
I_{\mathrm{o}}=I_{r}(x, y)+I_{m}(x, y) \cos (\Delta \Phi(x, y)) \\
I_{\pi / 2}=I_{r}(x, y)-I_{m}(x, y) \sin (\Delta \Phi(x, y)) \\
I_{\pi}=I_{r}(x, y)-I_{m}(x, y) \cos (\Delta \Phi(x, y))
\end{array}
$$

where $I_{r}(x, y)$ is the background intensity, $I_{m}(x, y)$ is the modulation intensity, and $\Delta \Phi(x, y)$ is the phase caused by the optical path difference between the reference beam and the object beams modulated by that due to the surface variation. Both $I_{r}(x, y)$ and $I_{m}(x, y)$ do not change significantly with the phase shifting. The intensities $I_{\mathrm{O}}$, $I_{\pi / 2}$ and $I_{\pi}$ are related to $\Delta \Phi(x, y)$ by:

$$
\tan \Delta \Phi(x, y)=\left(2 I_{\pi / 2}-I_{\mathrm{o}}-I_{\pi}\right) /\left(I_{\pi}-I_{\mathrm{o}}\right) .
$$

The surface variation of the object (gauge block) is determined by $(\lambda / 2 \pi) x \Delta \Phi(x, y)$. A simple Matlab program is used to evaluate the roughness from the intensities distributions of the three recorded interferograms (Fig. 5).

He-Ne laser of wavelength $632.8 \mathrm{~nm}$ is used and the intensities distributions are shown in Figure 6.

The evaluation of $\tan \Delta \Phi(x, y)$ and $\Delta \Phi(x, y)$ is shown in Figure 7, and gauge block surface profile is shown in Figure 8, and the measured value is found to be $0.03 \mu \mathrm{m}$.

In order to increase the accuracy of the technique, we advised a PZT of high resolution to allow phase shifting to $\pi / 5$, and this will be applied to the reference mirror of our suggested interferometer.

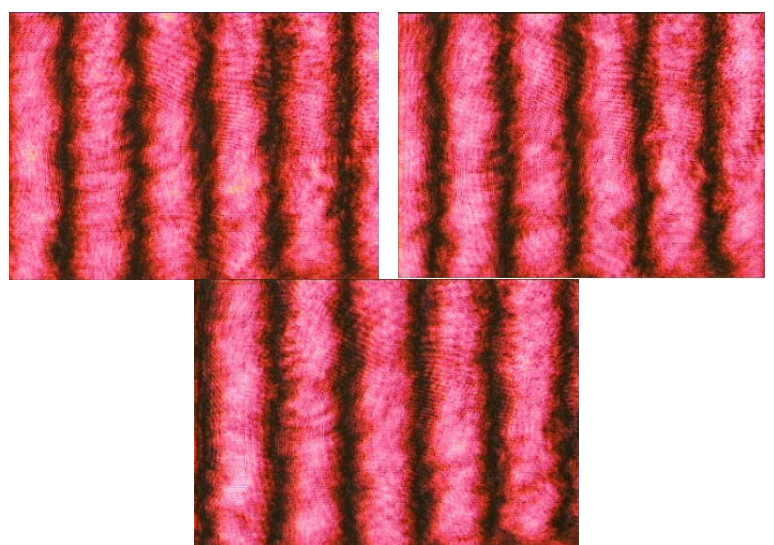

Fig. 7. Interference fringe at phase shifts: $o, \pi / 2$ and $\pi$.

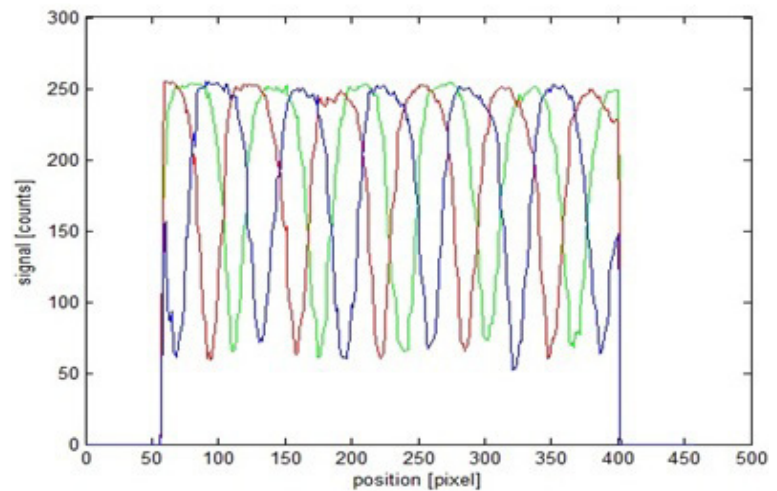

Fig. 8. Intensity distribution of the three interferograms.
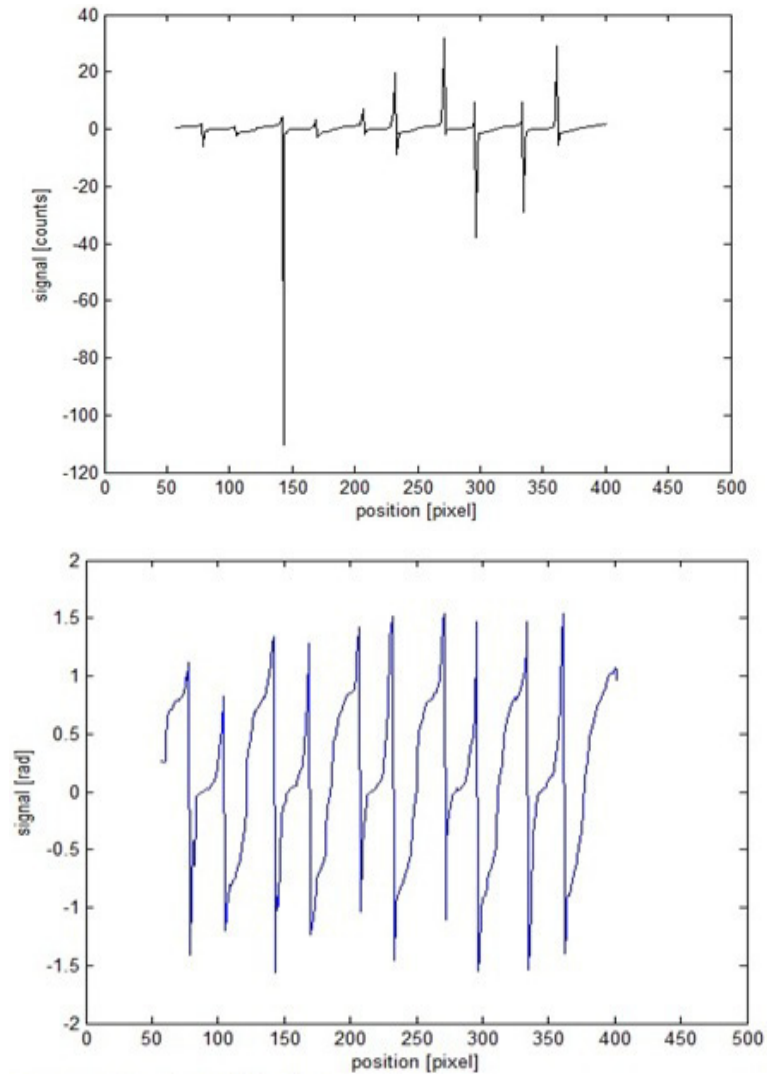

Fig. 9. The plot of: (a) $\tan \Delta \Phi(x, y)$ and (b) $\Delta \Phi(x, y)$. 


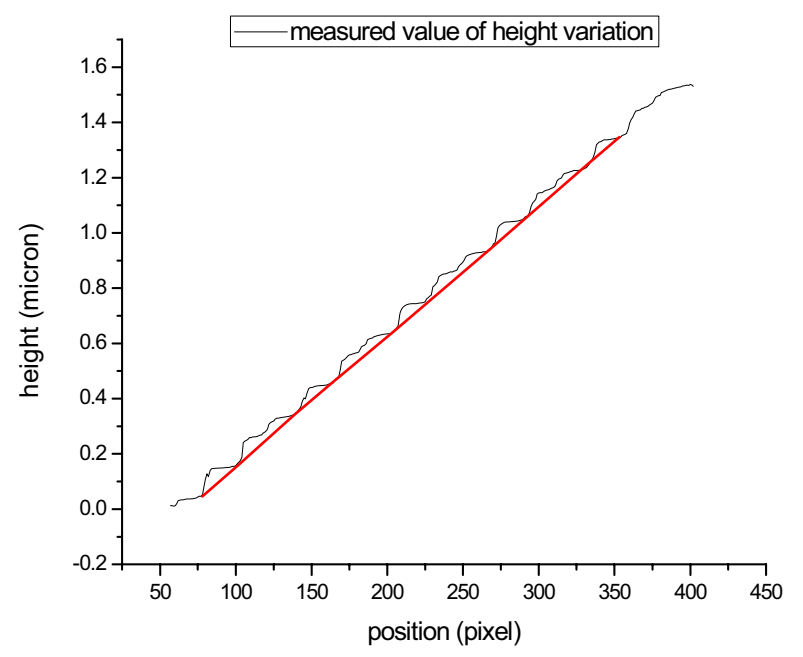

Fig. 10. Gauge block surface profile.

\section{Conclusion}

Due to the impact and the critical importance of end standards, both gauge blocks and length bars, NMISA and NIS took an decision to upgrade its current systems for the calibration of gauge blocks and length bars. A joint project between the NMIs from Egpyt, NIS and South Africa, NMISA were formed to reseasrh of different systems. For South Africa, the University of Stellenbosch joint the reasearch. NMISA and the university has a long relationships with many projects delivered on many areas of dimensional metrology. The university has especially good experince in the use of cameras and vision software which could be used in the fringes analises.

The paper at first investigated the current practise in the calibration of gauge blocks and length bars. Secondly the difficulties in the current practise of calibration systems.

Thridly, research in other systems where the use of double ended interfometery was investigated and two projects, one at each NMI were started.

The project is currently in its first year of a two year joint project between the organisations and experiemental setups and developement work will be performed over the next year, specially around the use of phase stepping, the use of the camrea to detect the fringes and the alingment of the gauge blocks relative to the laser beams. The project already shows promise to be in the futuue use for the use of the calibration of gauges blocks.

\section{References}

1. Y. Ishii, New method for interometric measurement of gauge blocks without wringing onto a platen. Metrologia 35, 67 (1998)

2. A. Abdelaty, A. Walkov, P. Franke, R. Schödel, Challenges on double ended gauge block interferometry unveiled by the study of a prototype at PTB, Metrologia 49, (2012)

3. ISO 3650:1998 (E), http://www.iso.org/obp/ui/\#iso: std: iso: 3650 : ed-2:v1:en

4. G.J. Siddal, P.C.T. Willey, Flat surface wringing and contact error variability, J. Phys. D 3, 8 (1970)

5. E.G. Thwaite, Phase correction in the interferometric measurement of end standards, Metrologia 14, 53-62 (1978)

6. K.V. Rajesh, A study of gage block wringing. Center for precision metrology, university of north Carolina at Charlotta, 2005. http://www . aspe.net/publications/ Annual_2005/POSTERS/3METRO/6SURF/1831.PDF

7. J.E. Dekker, P. Franke, G. Boensch, On the influence of gauge blocks roughness, Proc. SPIE 5879, 587907, 2005

8. A. Titov, I. Malinovsky, A. Massone, Gauge block measurements with nanometre uncertainty, Metrologia 37, 121 (2000)

9. A. Titov, I. Malinovsky, A. Massone, Realizing subnanometer accuracy level in gauge block measurements, Proc. SPIE 3477 (1998)

10. Bonsch, Concerning two recent papers on gauge block measurement by interferometry, Metrologia 39, 101 (2002)

11. Bonsch, Interferometric calibration of an integrating sphere for the determination of the roughness correction of gauge blocks, Proc. SPIE 3477, 152 (1998)

12. Tesa gauge block interferometer manual

13. O.W. Cramer, G. Thompson, R. Turner, An instrument for measuring end bars, S. Afr. J. Phys. 10, 1 (1987)

14. O. Kruger, T\&M conference, 2009

15. Z. Buchta, S. Řeřucha, B. Mikel, M. Č́žek, J. Lazar, O. Číp, Novel principle of contactless gauge block calibrations, Sensors 3, 3350-3358 (2012) 